\title{
Effect of Ring Machine Front Roller Rubber Cot Hardness on Properties of Coarser Yarn
}

\author{
Abdul Rehman Akbar ${ }^{1,2,3}$, Muhammad Zeshan³, Mohammad Neaz², Yingjie Cai ${ }^{1,4}$, and Lina Lin ${ }^{1,2,4, a}$ \\ ${ }^{1}$ Hubei Provincial Engineering Laboratory for Clean Production and High Value Utilization of Bio-based Textile Materials, Wuhan Textile \\ University, Wuhan 430200, China \\ ${ }^{2}$ College of Textile Science and Engineering, Wuhan Textile University, Wuhan 430200, China \\ ${ }^{3}$ Department of Yarn Manufacturing, National Textile University, Faisalabad 37250, Pakistan \\ ${ }^{4}$ Engineering Research Centre for Clean Production of Textile Dyeing and Printing, Ministry of Education, Wuhan Textile University, Wuhan \\ 430200, China
}

\begin{abstract}
The hardness of the rubber cot is one of the most important factors that influences the properties of spun cotton yarn. Four different 100\% coarse count cotton yarns (Ne 10/1, Ne 12/1, Ne 14/1, and Ne 16/1) were spun with various front roller rubber cot hardness from $65^{\circ}, 68^{\circ}, 70^{\circ}, 75^{\circ}$, and $83^{\circ}$ shore hardness with constant back cot roller shore hardness. Cotton yarn properties were investigated, including mass uniformity, imperfection level, unevenness, hairiness, tensile strength, and elongation. The results showed that as the shore hardness increased, the unevenness of mass and imperfection index of the yarns proportionally increased. It does not apparently influence the yarns' hairiness, tensile strength, or elongation properties.
\end{abstract}

\section{Introduction}

Yarn quality is essential for the economic success of spinning plants. International competition and market requirements dictate the necessity to produce quality yarns at an acceptable price. There have been numerous attempts to improve the yarn quality, including process modification, process optimization, and investigations [1]. The raw material, process control techniques, and spinning parameters are major factors that influence the final yarn properties [2-4]. Drafting is the most important and fundamental operation in ring spinning. It has substantial contribution to the yarn quality, particularly in spinning the top roller covers, i.e. cots and drafting aprons [5]. Cots and aprons in the drafting system contribute to the overall spinning performance and yarn quality, along with other components. Cots are used in draw frames, combers, speed frames, and ring frames. Aprons are used only in speed frames and ring frames. Cots work to provide uniform pressure on the fiber strand. This facilitates efficient drafting with the assistance of aprons, which results in a better grip and control of the fibers, particularly floating fibers. A front-line cot in the ring spinning also offers a sufficient pulling force. A minimum pulling force that is greater than or equal to the sum of frictional resistance between fibers and force exerted by the aprons on fibers is required at the front line cot to overcome drafting resistance. Special rubber compounds with shore hardness ranging from $63^{\circ}$ to $90^{\circ}$ are used as raw materials for the cots $[6,7]$. The raw material composition determines the properties of the cot.

\footnotetext{
a Corresponding author: linalin@wtu.edu.cn
}

This includes the shore hardness of the rubber cot, abrasion resistance, tensile strength, swelling resistance, resilience properties, surface characteristics (like grip offered on fiber strands), low compression set values, and elasticity of the cot and the color. The top roller cots should have good ageing stability, minimal film formation, no lap formation, good fiber guiding, and long working life for use as a top roller cover [8].

Synthetic top roller cots are typically available in cylindrical form. The length or width, the finished outer diameter, the bare roller diameter, and the construction like PVC (core and shore hardness) are technical requirements of a top roller cot. The degree of the shore hardness is a main property of the top roller cot. It varies within different types of fibers and applications [8]. Among the yarn quality parameters, hairiness is one of the most important factors in determining the quality of the spun yarn, or the fibers that protrude from the body of yarn as loops, wild fibers, and extra fiber ends [9]. The performance of the woven or the knitted fabric are greatly affected by these factors [10]. Hairiness can cause problems within machine stoppages and breakage during spinning, since it makes a weak point in the yarn. It can also affect the appearance, the pilling, and the handling of the fabric $[10,11]$. It also reduced the dye uptake of the fabric during dyeing [12]. This makes yarn hairiness a point of interest for researchers who aim to produce better quality yarn and fabric [10]. The imperfection index $(50 \%$ thick, $50 \%$ thin, and $200 \%$ nep) values, the uniformity of mass, the tensile strength, and the 
elongation of the yarn are important factors determining its quality and usage in the subsequent process [13].

\section{Experimental}

\subsection{Materials}

Yarns were made of cotton fibers. The cotton fiber had a strength of $30 \mathrm{~g} / \mathrm{tex}$, a Micronaire value of 4.5-5.0, a fiber elongation of $6.0 \%$, a tenacity of $3.0-4.9 \mathrm{~g} / \mathrm{Den}$, a moisture regain of $8.5 \%$, and a specific gravity of 1.54 .

\subsection{Procedure}

The production of the yarn samples was done with a Toyoda ring frame (Model: RX-240) auto-doffing linked with an auto-coner. Rubber cots with various hardness (range: $65^{\circ}, 68^{\circ}, 70^{\circ}, 75^{\circ}$ to $83^{\circ}$ ) shore were used on the front-line rollers in the RX-240 ring frame drafting system. The hardness of the back-line rollers in the drafting system were kept constant with a standard value of $83^{\circ}$ shore across all experiments. The other process parameters during the yarn production were kept constant at the opening machine, the cleaning machine, the carding machine, the breaker drawing machine, and the finisher drawing machine. The hank roving count if the simplex machine was changed to spin a specific count (yarn fineness). The vertical pneumatic mounting machine was used to mount the cots. The semi-automatic grinding machine was used to grind after the cots were mounted. A grinding stone with a stone grid of 80 grit size and a porosity of 14 was used. An average roughness value of $0.8 \pm 0.2 \mu \mathrm{m}$ was applied to both the front and back line cots within ring spinning. The yarn properties were analyzed after completion.

\subsection{Testing Instruments}

The unevenness of mass, the yarn hairiness (hair length greater and equal to $3 \mathrm{~mm}$ ), and the imperfection index of the yarn were determined with an USTER ${ }^{\circledR}$ evenness tester (UT-5, Uster Technologies AG, Switzerland). The yarn tensile strength properties were tested with a MESDAN lab strength tester (Italy).

\section{Results and discussion}

\subsection{Preparatory Process}

The cotton fibers need to be treated at different stages to make yarn. The fibers are treated on a card machine, a draw frame (breaker), a finisher draw frame, and a simplex before they are processed on the ring machine. These processes are called the preparatory processes. Each preparatory stage influences the quality of the final yarn. The fiber strand was tested after every stage to ensure the working conditions were suitable for producing good quality yarn. The unevenness percentage (U\%) of the semi-product at each stage were investigated, as shown in Figure 1.

The variation in mass per unit length of resultant yarn was caused by the variation in fiber assembly from the preparatory processes. The U\% of four different carded slivers were tested at UT-5. The mean value of the U\% measured is 2.78. The factors affected the $U \%$ of the fiber strand are the wire conditions of the machine parts and the setting of the machine parts with respect to each other. It can be controlled by using wires that are in its best working condition and proper settings of the machine. The U\% of four drawn slivers from different breaker draw frame machines is tested to check the quality of operation. The actual mean value of the unevenness comes out to be $2.88 \%$, which is less than the acceptable limit of $3 \%$. The unevenness depends upon drafting system; therefore, the optimum settings should be maintained in the drafting arrangement to get maximum uniformity in the product.

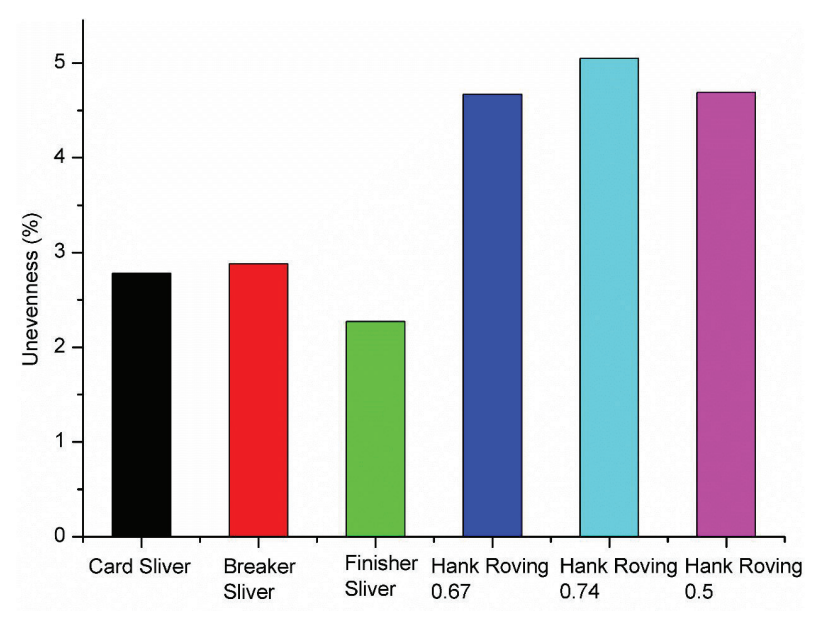

Figure 1. Unevenness after different processes during yarn spinning.

The finisher draw-frame is used to reduce the variations in the sliver. The finisher draw-frame has autoleveling system that helps to control the variations in the sliver. The sensing device i.e. tong and groove roller checks the variations, and the signals are given back to control the variations. The mean value of $\mathrm{U} \%$ for four samples is 2.26 that is good as compared to the values of breaker sliver.

In processing in the spinning mill, the unevenness of the product increases from stage to stage after draw frame [15]. Four different samples of roving with same hank roving from four different simplex machines have been tested. The actual mean value of the U\% obtained is 4.67 . The variation may arise due to the setting problems between the rollers, draft distribution, parts used within the drafting arrangement and some other factors. The U\% is one of the important factor determining the quality of the product being produced on the machine. The lower it is, better the quality and vice versa. In order to get the best results out of the simplex machine all factors that affect quality should be taken into account.

Another parameter that determine the quality of the product being produced is coefficient of variation of mass $(\mathrm{CVm} \%)$. Co-efficient of variation in the semi-product should be within acceptable range to get a product with desired properties and quality. Higher $\mathrm{CVm}$ in the yarn means lower product quality. Generally during spinning, the $\mathrm{CVm} \%$ of the fiber strand decreases from carding to finisher but it drastically increases at simplex. The 
$\mathrm{CVm} \%$ of the fiber strands were noted after each step. The mean value for the four samples were taken at each stage. The results are shown in Figure 2.

The coefficient of the mass variation $(3.6 \%)$ was measured for the slivers after the carding operation. The variation reduced further to $3.4 \%$ after the first draw frame passage, and then again to $2.9 \%$ after the material passed through the finisher draw frame. The step wise decrease in mass variation was due to doubling that occurred at the draw frame. Doubling refers to adding additional slivers (6 to 8 typically) to obtain an additional equalized sliver after drafting [14]. The CVm of the fiber strand was also checked after processing through a simplex machine. The obtained value was approximately $6 \%$. Simplex attenuated the fiber strand obtained the desired roving counts (i.e. 0.67, 0.74, and 0.5 hank roving).

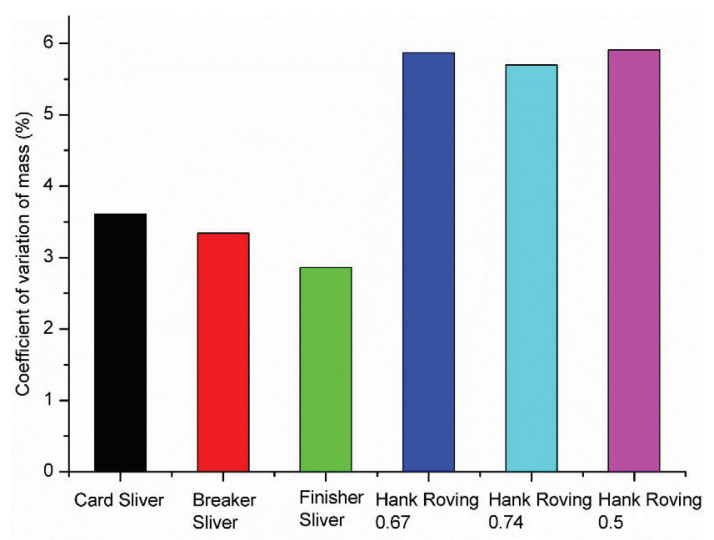

Figure 2. The coefficients of variation after various processes during yarn spinning.

\subsection{Influence of rubber cots hardness on yarn unevenness of mass}

Mass uniformity is required for good quality yarn [14]. The unevenness of the mass increase with the increase of rubber cots shore hardness are shown in Figure 3. This is particularly apparent for the 14/1 and 16/1 count yarn (fineness of single yarn). The mean values of the unevenness were $10.5 \%, 10.62 \%, 10.84 \%, 10.92 \%$, and $11.32 \%$ for $\mathrm{Ne} 14 / 1$ and $\mathrm{Ne} 16 / 1$. The hardness shore was $65^{\circ}, 68^{\circ}, 70^{\circ}, 75^{\circ}$, and $83^{\circ}$. The lower shore hardness gave more control of the fibers, due to an increased contact area between the front roller cot and the bottom fluted rollers. The increased contact area shortened the uncontrolled length of the fiber strand between the cot nipping point and the apron. Therefore, softer cots produce more even yarn [8]. Note, the highest uniformity of yarn mass occurred at $68^{\circ}$ shore of the cot hardness.

\subsection{Influence of rubber cots hardness on imperfection Index}

The imperfection index values (provided in Figure 4) depicts the directly proportional relationship with the cots hardness. This indicated that softer cots produced a lower imperfection index of yarn. The mean values of the imperfection index for the four different yarn counts were $86.45 \%, 100.23 \%, 119.15 \%, 122.8 \%$, and $149.77 \%$ with a shore hardness of $65^{\circ}, 68^{\circ}, 70^{\circ}, 75^{\circ}$, and $83^{\circ}$. This was attributed to the decrease in the uncontrolled length between the aprons and the nipping point of the front roller cots while using softer cots. This led to a more controlled flow of the material. The uncontrolled length was the length where the material had no support to be carried. The fiber strands then moved and slid apart during drafting [15]. This uneven flow of material was reduced by the decreased uncontrolled length using relatively softer cots. The softer cots shift the nipping point of the front rubber rollers slightly forward, due to the increased cross-sectional contact. It reduced the length of the spinning triangle, which caused the twist to be inserted in a better way for the fiber strand to exit the rollers. The improved twist realization led to an improved imperfection index [8].

The yarn quality with lower imperfection index values were achieved by having less co-efficient of variation of mass and good mass uniformities, resilience property and low compression set values, and anti-lapping characteristics. These played a crucial role, since they affected the spinning performance. The lapping characteristics were important for the rubber cots because they influenced the quality of the product and the performance of the operation. A majority of the lapping originated after an end break. The finer fibers were more likely to follow the profile of the roller and had more of a lapping tendency. The finer the fibers, the more likely they were in the cross-section of the yarn. This led to an increased frequency of lapping. The applied pressure on the top rollers also influenced the lapping behavior of the fibers, resulting in a deterioration of the quality. Finer and longer fibers had more of a tendency to lap, since more pressure was needed to overcome the drafting resistance during spinning. Pneumatic suction is another parameter that was affected. The lower values of suction pressure had an increased tendency of lapping and vice versa. It could be adjusted by varying the different parameters, such as $\backslash$ fan size, speed, and machine length. The mini pores, or pinholes in the rubber cots or impurities in the cots, also caused lapping. Softer rubber cots had more of a tendency for lapping, due to increased contact area. Therefore, frequent buffing of the cots (every 30 days) and after treatment could improve the quality of the process and the product.

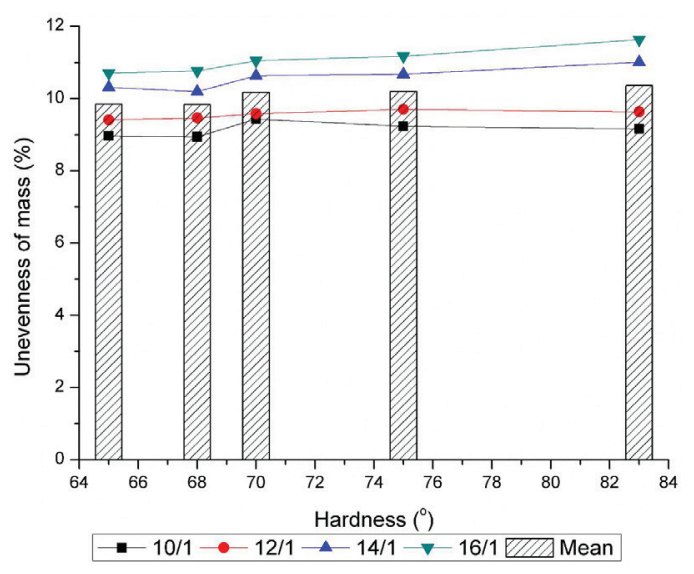

Figure 3. Unevenness of the mass of yarns spun with various rubber cots shore hardness at $65^{\circ}, 68^{\circ}, 70^{\circ}, 75^{\circ}$, and $83^{\circ}$ shore. 


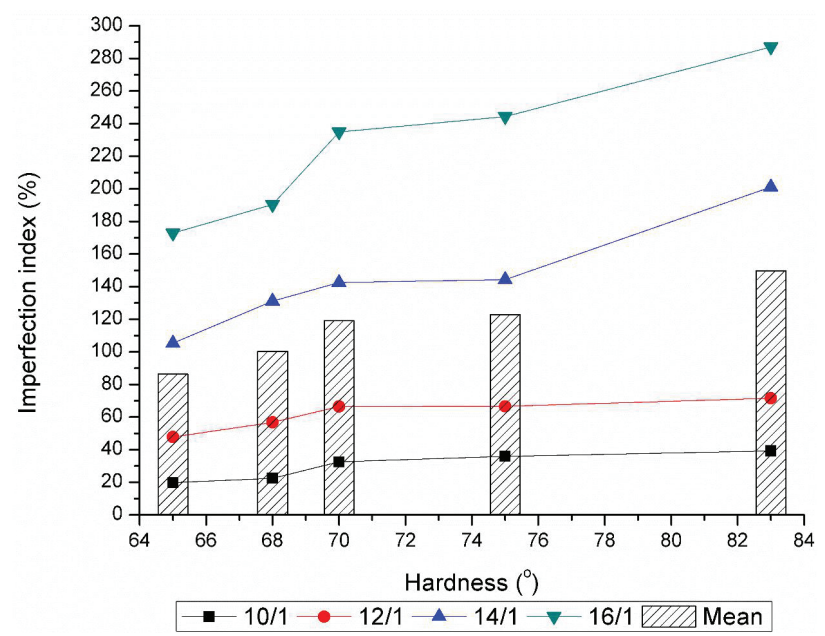

Figure 4. Imperfection index of yarns spun with various rubber cots shore hardness at $65^{\circ}, 68^{\circ}, 70^{\circ}, 75^{\circ}$, and $83^{\circ}$ shore.

\subsection{Influence of rubber cots hardness on hairiness}

Figure 5 summarizes the hardness levels of the rubber cots and the variation in the yarn hairiness. Rubber cots with a shore hardness of $68^{\circ}$ was considered ideal for ring spinning. Our results confirmed this finding. The $70^{\circ}$ shore hardness had the highest hairiness out of all the counts. There was no specific trend for the increase or the decrease in hairiness values with variation of the rubber cot hardness. There should be an optimum level of hardness to achieve lowest hairiness [16].

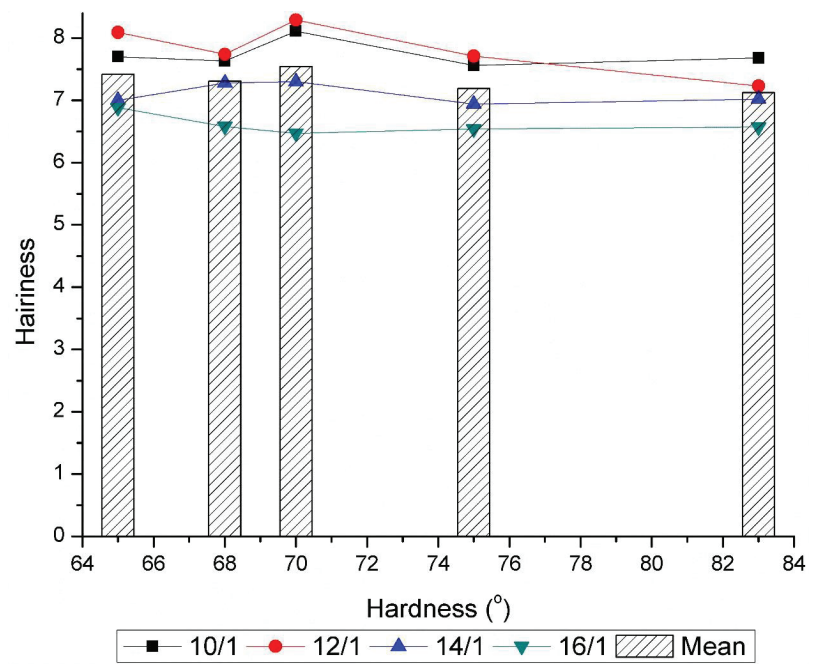

Figure 5. The hairiness of the yarns spun with various rubber cots shore hardness of $65^{\circ}, 68^{\circ}, 70^{\circ}, 75^{\circ}$, and $83^{\circ}$ shore.

The hairiness increased at two points during ring spinning, i.e. the front roller delivery point and the ring traveler junction. The twist did not flow directly to the nip point of the spinning triangle. The selvedge fibers in the strand did not fully integrate into the yarn. They appeared as hairs. An abrasion at the traveler/ring junction led to portions of the fibers to travel into hairs. The count and the twist had a considerable influence on the hairiness. The courser count yarns had increased amounts of hairiness than the finer count yarns because they had more cross-sectional area. The proportion of fiber ends that protruded from the yarn surface were about $31 \%$ of the actual number of ends present in the yarn. This was measured microscopically. The mean value of the hairiness increased as the cross-sectional area increased when the length of the protruding fiber ended and the loops were considered. An optimum value of hardness was necessary, as harder rubber cots damaged the fibers when the high compression increased the hairiness breakage occurred. The lower rubber hardness lapped the fibers around the cots, which led to breakage and low yarn quality [8].

\subsection{Influence of rubber cots hardness on tensile strength and elongation}

Tensile testing of the yarns was performed to determine the breaking force, elongation, and toughness properties. Tensile testing of yarn was performed by a tensile testing machine that used a unique grip to hold the yarn. Resistance against the pulling force was referred as the tensile strength $[4,17,18]$. This is an important characteristic of the yarn, which influenced its processing quality in the subsequent processes. The yarn should have an optimum level of tensile strength with optimum elongation. The influence of the changes in hardness values of the front roller rubber cots over the tensile strength and the elongation values are shown in Figure 6. There was no specific pattern found between the values of tensile strength and elongation with various hardness levels. The increased rubber cot hardness did not have an apparent direct influence on the yarn tensile strength and elongation properties. The mean values were approximately $211 \mathrm{lbs}$. (219 lbs. for $68^{\circ}$ shore) and $13 \%$ under the various hardness conditions. The coarse yarn properties analysis showed that the optimum rubber cot hardness during ring spinning was $68^{\circ}$ shore $[8,19]$.

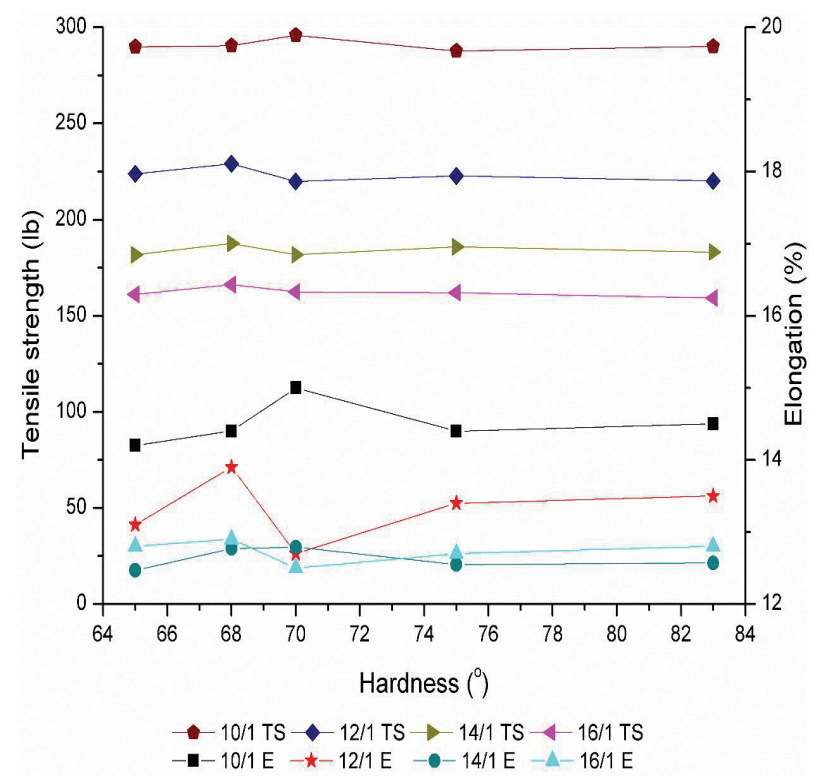

Figure 6. The tensile strength and the elongation of yarns spun with various rubber cots shore hardness at $65^{\circ}, 68^{\circ}, 70^{\circ}, 75^{\circ}$, and $83^{\circ}$ shore. 


\section{Conclusion}

Four different $100 \%$ coarser count cotton yarns (Ne 10/1, $\mathrm{Ne} 12 / 1, \mathrm{Ne} 14 / 1$, and $\mathrm{Ne} 16 / 1)$ were spun with rubber cots of various hardness. These ranged from $65^{\circ}, 68^{\circ}, 70^{\circ}$, $75^{\circ}$, to $83^{\circ}$ shore on the front-line rollers. As hardness increased, the unevenness of the mass and the imperfection index of yarns proportionally increased. For hardness ranging $65^{\circ}, 68^{\circ}, 70^{\circ}, 75^{\circ}$, and $83^{\circ}$ shore, the average values for four yarn counts were $9.8 \%, 9.8 \%$, $10.2 \%, 10.2 \%$, and $10.4 \%$. The imperfection index mean values for four counts were $86.5 \%, 100.2 \%, 119.2 \%$, $122.8 \%$, and $149.8 \%$. The increase of rubber cot hardness did not have an apparent, direct influence on the properties of yarn hairiness, tensile strength, or elongation. The mean values were approximately $7 \mathrm{lbs}$, $116 \mathrm{lbs}$. (220 lbs. for $68^{\circ}$ shore) and $13 \%$ under the varied hardness conditions. According to the analysis of the coarse yarn properties, the optimum rubber cot hardness that should be applied in ring spinning was $68^{\circ}$ shore.

\section{Acknowledgements}

This work was financially supported by the China National Textile \& Apparel Council (2013 "Textile Vision" Applied Basic Research, 2013-153); Hubei Province Science and Technology Support Program (Grant No. 2013BAA043) and the Collaborative Innovation Plan of Hubei Province for Key Technology of Eco-Ramie Industry (2014-8).

\section{References}

1. N. Balasubramanian, Text. Res. J. 39, 155 (1969)

2. P.K. Majumdar, A. Majumdar, Text. Res. J. 74, 652 (2004)

3. P.R. Lord, Handbook of Yarn Production: Technology, Science and Economics (Elsevier, 2003)

4. C.A. Lawrence, Fundamentals of Spun Yarn Technology (CRC Press, London, 2003)

5. S. Sundaresan, A. Arunraj, K. Thangamani, International Journal of Innovative Research in Science, Engineering and Technology 5, 2026 (2016)

6. G. Grover, P.R. Lord, J. Text. Inst. 83, 560 (1992)

7. V. Subramaniam, A.P. Mohamed, Text. Res. J. 61, 280 (1991)

8. A.S. Bagwan, R. Policepatil, S. Pawar, J. Text. Sci. Eng. 6, 238 (2016)

9. X.X. Huang, X.M. Tao, B.G. Xu, Text. Res. J. 84, 1121 (2014)

10. N. Haleem, X. Wang, Text. Res. J. 85, 211 (2015)

11. Z. Xia, X. Wang, W. Ye, W. Xu, H.A. Eltahir, Fiber Polym. 12, 534 (2011)

12. X. Wang, L. Chang, Text. Res. J. 73, 327 (2003)

13. R. Furter, The Standardization of the Quality Characteristics in the Textile Supply Chain, Uster Technologies AG, (2009)

14. W. Klein, The Rieter Manual of Spinning. Volume 1. Technology of Short-Staple Spinning, Winterthur: Rieter Machine Works Ltd, (2008)
15. W. Klein, H. Stalder, The Rieter Manual of Spinning.Volume 4. Ring Spinning, (Rieter Machine Works, Winterthur, 2008)

16. A. Barella, Text. Prog. 13, 1 (1983)

17. E. Oxtoby, Spun Yarn Technology (ButterworthHeinemann, London, 2013)

18. C. Horst, S. Tetsuya, S. Leslie, Handbook of Materials Measurement Methods (Springer, Berlin, 2006)

19. V. Subramaniam, A.P. Mohamed, J. Text. Inst., 82, 333 (1991) 